\title{
Modelado del Efecto de la Temperatura, Actividad de Agua y pH sobre el Crecimiento de Rhizopus oryzae
}

\author{
M.L. Carrillo*, D. Zavala y B. Alvarado \\ Universidad Autónoma de San Luis Potosí, Unidad Académica Multidisciplinaria Zona Huasteca, \\ Calle Romualdo del Campo No 501, Fracc. Rafael Curiel, San Luis Potosí-México \\ (e-mail: maluisa@uaslp.mx)
}

* autor a quien debe ser dirigida la correspondencia

\begin{abstract}
Resumen
El objetivo de este trabajo fue modelar el crecimiento de Rhizopus oryzae, hongo deteriorativo de alimentos, en función de la temperatura, la actividad de agua $\left(a_{w}\right)$ y el $\mathrm{pH}$. Las curvas de crecimiento de Rhizopus oryzae se generaron a las condiciones de temperatura (20 y $\left.30^{\circ} \mathrm{C}\right), \mathrm{a}_{\mathrm{w}}(0.895$ y 0.99$)$ y $\mathrm{pH}$ (3 y 5) dadas por un diseño factorial completo. Dichas curvas se obtuvieron al graficar el diámetro de las colonias contra el tiempo y se ajustaron con el modelo de Baranyi para obtener los parámetros de crecimiento (velocidad específica de crecimiento, tiempo de latencia y crecimiento máximo), a partir de los cuales se generó un modelo polinomial. El análisis estadístico indicó que todos los factores ejercen un efecto significativo $(p<0.001)$ sobre el crecimiento del hongo.
\end{abstract}

Palabras clave: Rhizopus oryzae, modelo de Baranyi, hongos deteriorativos, crecimiento de hongos

\section{Modeling the Effects of Temperature, Water Activity and pH on the Growth of Rhizopus oryzae}

\begin{abstract}
The proposal of this work was modeling the growth of Rhizopus oryzae- fungus deteriorative food- in function of temperature, water activity $\left(a_{w}\right)$ and $\mathrm{pH}$. Growth curves of $R$. oryzae were generated at conditions of temperature $\left(20\right.$ and $\left.30^{\circ} \mathrm{C}\right), \mathrm{a}_{\mathrm{w}}(0.895$ and 0.99$)$ and $\mathrm{pH}(3$ and 5$)$ giving in factorial full design. Such curves were obtained to graphic the colony diameter versus time and were adjusted with the Baranyi model in order to obtain the growth parameters - specific growth rate, latency of time and maximal growth-, to left was generated a polynomial model. The three factors were statistically significant $(p<0.001)$ on the fungi growth.
\end{abstract}

Keywords: Rhizopus oryzae, Baranyi model, deteriorative fungi, growth of fungi 


\section{INTRODUCCIÓN}

Una de las causas del deterioro de los alimentos es la actividad bioquímica resultante de la multiplicación de microorganismos específicos, por lo que existe la necesidad de comprender los factores que controlan el crecimiento de microorganismos en los alimentos.

Para determinar el efecto de los factores de conservación: temperatura, actividad de agua $\left(\mathrm{a}_{\mathrm{w}}\right)$ y $\mathrm{pH}$ sobre el crecimiento microbiano, se usa como una herramienta útil la microbiología predictiva (Dens et al., 1999), que es un área de investigación de la microbiología en la que se combinan el conocimiento microbiano y matemático para desarrollar modelos que describan la evolución microbiana. Así, es posible predecir el desarrollo de un microorganismo de interés en base a una relación matemática entre las respuestas del crecimiento microbiano y las condiciones ambientales (Vereecken et al., 2000; Doona et al., 2004).

Entre los microorganismos más estudiados se encuentran las bacterias, sin embargo, actualmente se ha incrementado el interés por describir el crecimiento de los hongos, agentes causales de la descomposición de alimentos (Jay, 2002), uno de ellos es Rhizopus oryzae, que se ha aislado de suelos, cereales, agua contaminada y vegetales, el cual es de amplia distribución mundial, pero crece principalmente en zonas tropicales y subtropicales (Samson et al., 1995). Las condiciones óptimas de crecimiento para $R$. oryzae se reportan a $\mathrm{T}=30^{\circ} \mathrm{C}, \mathrm{a}_{\mathrm{w}}=0.9 \mathrm{y} \mathrm{pH}=3.0$ (Halfpap, 2005), $\sin$ embargo, al emplear simultáneamente varios factores de conservación, el nivel óptimo de cada uno de éstos puede variar; de ahí la importancia de estudiar el efecto de dichos factores en forma combinada. Por lo anterior, este trabajo tuvo como objetivo describir matemáticamente el efecto de la temperatura, $\mathrm{a}_{\mathrm{w}} \mathrm{y} \mathrm{pH}$ sobre el crecimiento de Rhizopus oryzae.

\section{MATERIALES Y MÉTODOS}

Para cumplir con los objetivos propuestos se empleó un diseño factorial completo considerando valores de temperatura, $\mathrm{a}_{\mathrm{w}} \mathrm{y} \mathrm{pH}$ en el rango en que se desarrolla el Rhizopus oryzae (Adams y Moss, 1997; Frazier y Westhoff, 2000).

En los experimentos realizados se utilizó una cepa de $R$. oryzae aislada de vegetales en descomposición e identificada de acuerdo a la descripción de Samson et al. (1995). La cepa aislada se inoculó en cuñas de agar dextrosa Saboraud (ADS) y se incubó a $25^{\circ} \mathrm{C}$ por 7 días. Por medio del lavado de la superficie de las cuñas de agar con $10 \mathrm{~mL}$ de Tween 80 al $0.1 \%$ (Shapira et al., 1996) se obtuvo una suspensión de aproximadamente $10^{6}$ esporas $/ \mathrm{mL}$ (contando en un hematocitómetro) (Meletiadis et al., 2001), que se usó inmediatamente.

Como medio de cultivo se empleó ADS (Bioxon). El pH de los medios se ajustó con una solución de ácido cítrico (Reactivos Monterrey) al $10 \%(\mathrm{p} / \mathrm{v})$, la cual se esterilizó con filtros de membrana de poro de $0.45 \mu \mathrm{m}$. La $\mathrm{a}_{\mathrm{w}}$ de los medios de cultivo se ajustó mediante la adición de sacarosa (Hycel). La cantidad de sacarosa a utilizar en cada condición fue calculada por medio de las ecuaciones de Ross (1975, ecuación 1) y Norrish (1966, ecuación 2) que se muestran a continuación.

\section{Ecuación de Ross:}

$a_{w f}=a_{w 1} \cdot a_{w 2}$

donde $a_{w f}$ es la actividad de agua final deseada del medio de cultivo, $a_{w 1}$, es la actividad de agua del medio de cultivo y $a_{w 2}$ es la actividad de agua del medio con sacarosa.

\section{Ecuación de Norrish:}

$a_{w}=x_{w} \exp \left[-k x_{s}^{2}\right]$ 
donde $a_{w}$ es la actividad de agua del medio de cultivo, $x_{w}$ es la fracción molar del agua, $x_{s}$ es la fracción molar del soluto y $k$ es la constante para el soluto.

La actividad de agua de los medios de cultivo se comprobó con un instrumento Aqualab CX2 de punto de rocío (Decagon Devices, Pullman, Wash).

Posteriormente se inocularon por duplicado placas de ADS, se incubaron a las temperaturas del diseño y diariamente se midió el crecimiento del hongo a partir del diámetro de las colonias.

Las curvas de crecimiento de los hongos, obtenidas al graficar el diámetro de las colonias contra el tiempo, se ajustaron con el programa DMFit, que emplea el modelo de Baranyi (Baranyi y Roberts, 1994, ecuación 3), para determinar los parámetros de crecimiento -velocidad de crecimiento, tiempo de latencia y crecimiento máximo- en cada una de las condiciones probadas.

El modelo de Baranyi ha sido usado para ajustar las curvas de crecimiento comúnmente observadas en bacterias: curvas lineales, aquellas con fase de latencia, aquellas con fase de muerte o declinación y curvas sigmoides (Xiong et al., 1999). El crecimiento de los hongos presenta también esta diversidad de curvas de crecimiento, y el modelo de Baranyi ha sido usado también para ajustar las curvas de crecimiento de estos microorganismos (Meletiadis et al., 2001; Panagou et al., 2003). Y aunque el diámetro de las colonias no necesariamente representa la verdadera naturaleza del crecimiento de los hongos, es la forma más simple y directa de medir el crecimiento (Gibson y Hocking, 1997).

$$
\begin{aligned}
& y(t)=y_{0}+\mu_{\max } t+\frac{1}{\mu_{\max }} \ln \left(e^{-v \cdot t}+e^{-h_{0}}-e^{-v \cdot t-h_{0}}\right) \\
& -\frac{1}{m} \ln \left(1+\frac{e^{m \mu_{\max } t+\frac{1}{\mu_{\max }} \ln \left(e^{-v \cdot t}+e^{-h_{0}}-e^{-v \cdot t-h_{0}}\right)}-1}{e^{m\left(y_{\max }-y_{0}\right)}}\right)
\end{aligned}
$$

donde $y(t)$ es la concentración celular o el diámetro de las colonias, $y_{0}$ es la concentración o diámetro inicial, $\mu_{\max }$ es la velocidad específica de crecimiento $(1 / h), m$ es un parámetro de curvatura para caracterizar la transición de la fase exponencial, $v$ es un parámetro de curvatura para caracterizar la transición a la fase exponencial y $h_{0}$ es un parámetro adimensional que cuantifica el estado fisiológico inicial de las células. De donde el tiempo de latencia $(h)$ es calculado como $h_{0} / \mu_{\max }$.

Una vez obtenidos los parámetros, se empleó el programa Statistica ${ }^{\mathrm{TM}}$ (Statsoft ${ }^{\mathrm{TM}}$, Tulsa, OK).para generar un modelo matemático que describe el cambio de cada parámetro de crecimiento en función los factores de conservación estudiados.

\section{RESULTADOS Y DISCUSIÓN}

Las curvas de crecimiento mostraron diferentes formas en cada condición (Figura 1) lo que se debió a la manipulación de los diferentes factores de conservación.

Con respecto a los parámetros de crecimiento, la mayor velocidad específica de crecimiento fue de $0.63 \mathrm{~h}^{-1}$ y se observó a una $\mathrm{T}: 20^{\circ} \mathrm{C}, \mathrm{a}_{\mathrm{w}}: 0.895 \mathrm{y} \mathrm{pH}: 5$. El tiempo de latencia y el crecimiento máximo 
en esa misma condición, fue de 92.52 h y $85.77 \mathrm{~mm}$ respectivamente (Tabla 1). Puede observarse que aunque el hongo creció a una mayor velocidad presentó un tiempo de latencia mayor al de otras condiciones, lo que se explica por el hecho de que la velocidad de crecimiento es una característica intrínseca de los microorganismos, mientras que el tiempo de latencia y el crecimiento máximo, son más susceptibles a la acción de factores ambientales (Baranyi y Roberts, 1994).

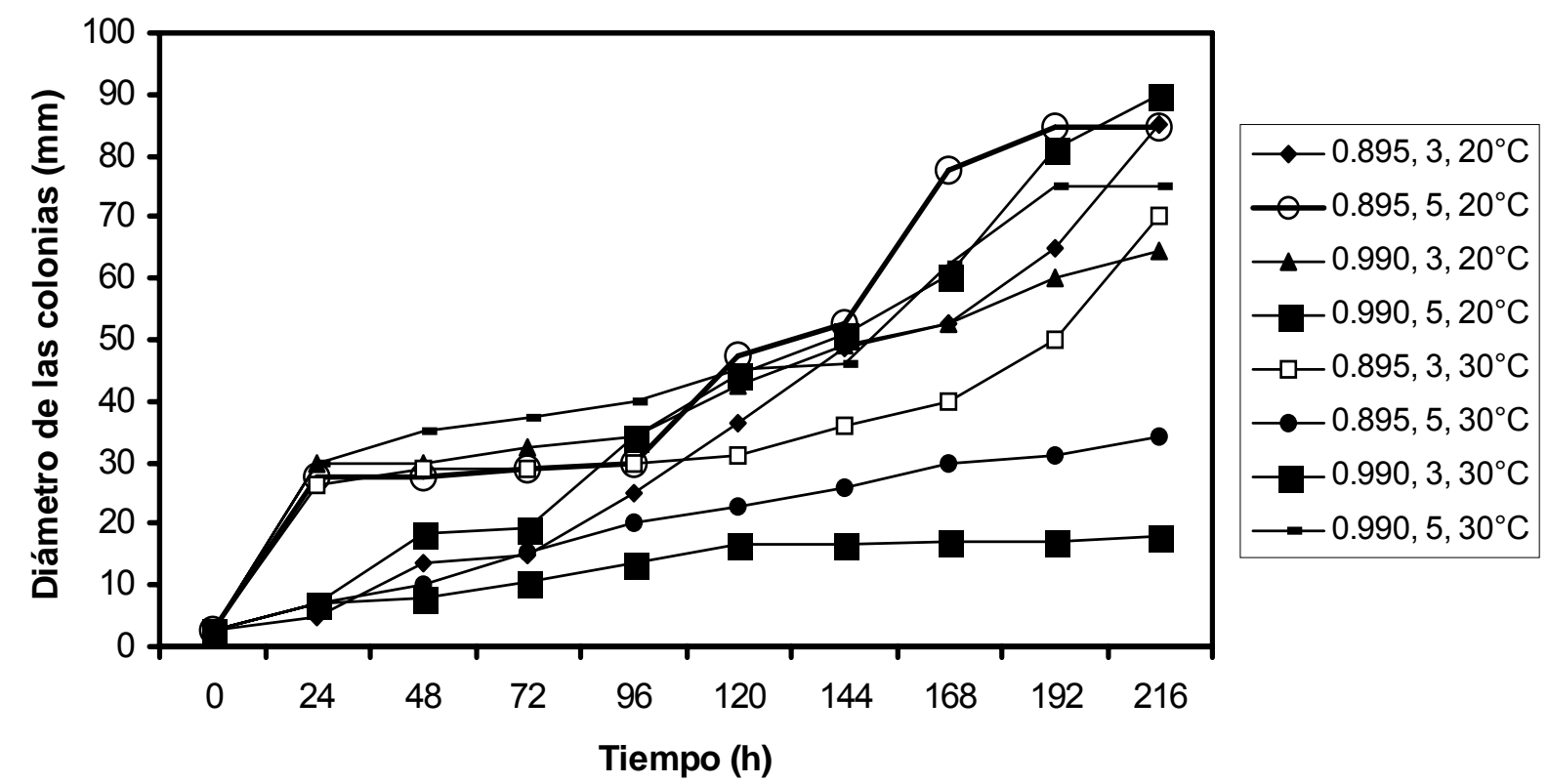

Fig. 1: Curvas de crecimiento de Rhizopus oryzae obtenidas a diferentes condiciones de temperatura, $\mathrm{a}_{\mathrm{w}} \mathrm{y} \mathrm{pH}$.

Tabla 1: Parámetros de crecimiento-velocidad de crecimiento $(\mu)$, tiempo de latencia $(\lambda)$ y crecimiento máximo ( $\left.y_{\text {máx }}\right)$ de Rhizopus oryzae a diferentes condiciones ( ${ }^{*}$ Desviación estándar)

\begin{tabular}{|c|c|c|c|c|c|}
\hline \multicolumn{2}{|c|}{ Factor } & \multicolumn{4}{|c|}{ Parámetros de crecimiento } \\
\hline $\mathrm{T}\left({ }^{\circ} \mathrm{C}\right)$ & $a_{w}$ & $\mathrm{pH}$ & $\begin{array}{c}\mu\left(\mathrm{h}^{-1}\right) \\
\left(^{*}\right)\end{array}$ & $\begin{array}{c}\lambda(\mathrm{h}) \\
\left(^{*}\right)\end{array}$ & $\begin{array}{c}y_{\text {máx }}(\mathrm{mm}) \\
\left(^{*}\right)\end{array}$ \\
\hline 20 & 0.895 & 3 & $\begin{array}{r}0.46 \\
\pm 0.05\end{array}$ & $\begin{array}{l}59.93 \\
\pm 2.52\end{array}$ & $\begin{array}{r}85 \\
\pm 1.26\end{array}$ \\
\hline 20 & 0.895 & 5 & $\begin{array}{r}0.63 \\
\pm 0.01\end{array}$ & $\begin{array}{l}92.52 \\
\pm 5.76\end{array}$ & $\begin{array}{l}85.77 \\
\pm 3.87\end{array}$ \\
\hline 20 & 0.990 & 3 & $\begin{array}{r}0.23 \\
\pm 0.19\end{array}$ & $\begin{array}{l}60.64 \\
\pm 3.25\end{array}$ & $\begin{array}{r}64.5 \\
\pm 1.23\end{array}$ \\
\hline 20 & 0.990 & 5 & $\begin{array}{r}0.46 \\
\pm 0.03\end{array}$ & $\begin{array}{r}36 \\
\pm 1.15\end{array}$ & $\begin{array}{r}90 \\
+2.07\end{array}$ \\
\hline 30 & 0.895 & 3 & $\begin{array}{r}0.59 \\
\pm 0.02\end{array}$ & $\begin{array}{r}144.53 \\
\pm 3.5\end{array}$ & $\begin{array}{r}70 \\
+2.78\end{array}$ \\
\hline 30 & 0.895 & 5 & $\begin{array}{r}0.18 \\
\pm 0.01\end{array}$ & $\begin{array}{r}10 \\
\pm 0.95\end{array}$ & $\begin{array}{l}31.89 \\
\pm 2.35\end{array}$ \\
\hline 30 & 0.990 & 3 & $\begin{array}{r}0.16 \\
\pm 0.03\end{array}$ & $\begin{array}{r}2.81 \\
\pm 0.09\end{array}$ & $\begin{array}{l}24.81 \\
\pm 1.10\end{array}$ \\
\hline 30 & 0.990 & 5 & $\begin{array}{r}0.27 \\
\pm 0.11\end{array}$ & $\begin{array}{r}40.47 \\
\pm 1.65\end{array}$ & $\begin{array}{r}75 \\
\pm 3.45\end{array}$ \\
\hline
\end{tabular}

Una vez obtenidos los parámetros de crecimiento, se generaron modelos polinomiales -utilizando el inverso de la velocidad de crecimiento y el inverso del tiempo de latencia con la finalidad de disminuir 
la varianza (McMeekin et al., 1993) y evitar valores negativos de tiempo de latencia-, para cada uno de los parámetros, cuyos coeficientes se muestran en la Tabla 2. El análisis estadístico mostró que los factores empleados tienen efecto significativo $(p<0.0001)$ en el crecimiento del hongo.

La descripción matemática del efecto de factores de conservación y su interacción sobre el crecimiento del hongo, es importante debido a que los modelos generados contribuyen a una mejor comprensión de la ecología microbiana de los alimentos.

Las interacciones entre los factores de conservación modifican los parámetros de crecimiento del hongo, siendo la temperatura el factor que más afecta el crecimiento de Rhizopus oryzae, seguido por la actividad de agua y en menor intensidad por el $\mathrm{pH}$.

Tabla 2. Coeficientes de regresión de los factores e interacciones de los modelos polinomiales para los parámetros de crecimiento de $R$. oryzae.

\begin{tabular}{lrrr}
\hline \multicolumn{5}{c}{ Modelo } \\
$\beta_{6} * a_{w} * p H$ \\
\multicolumn{5}{c}{$1 / \mu$} & & $1 / \lambda$ & & \\
\hline$\beta_{0}$ & -36.18 & -10.06 & 1395.84 \\
$\beta_{1}$ & -1.12 & 0.09 & -7.90 \\
$\beta_{2}$ & 46.16 & 10.63 & -1384.10 \\
$\beta_{3}$ & 10.99 & 1.95 & -262.82 \\
$\beta_{4}$ & 1.08 & -0.09 & 6.46 \\
$\beta_{5}$ & 0.05 & 0.01 & -0.31 \\
$\beta_{6}$ & -13.48 & -2.16 & 292.33 \\
\hline
\end{tabular}

\section{CONCLUSIONES}

La combinación de factores de conservación es determinante para los parámetros de crecimiento de los hongos deteriorativos de alimentos; una ligera variación en alguno de ellos constituye la diferencia entre la conservación y la descomposición de un alimento por cuestiones microbiológicas.

El crecimiento de Rhizopus oryzae, se ve favorecido a una temperatura de $20^{\circ} \mathrm{C}$, una $\mathrm{a}_{\mathrm{w}}$ de 0.895 y $\mathrm{pH} 5$, condiciones que deben evitarse en un alimento, con la finalidad de evitar se deterioro.

\section{REFERENCIAS}

Adams, M.R. y M.D. Moss; “Microbiología de los Alimentos”, p 24, Ed. Acribia. Zaragoza, España (1997).

Baranyi, J. y T.A. Roberts; A dynamic approach to predicting bacterial growth in food, Int. J. Food Microbiol., 23, 277- 294 (1994).

Dens, E.J., K.M. Vereeken y J.F. Van Impe; A prototype model structure for mixed microbial populations in homogeneus food products, J. Theor. Biol., 201, 159-170 (1999).

Doona, C. J., F.E. Feeherry, y E.W. Ross; A quasi-chemical model for the growth and death of microorganisms in foods by non thermal and high pressure processing, Int. J. Microbiol., 10 (1-3), 2132 (2004). 
Halfpap, F.D.; Efeito da temperatura na cinética de crescimiento de Rhizopus oryzae em cultivo no estado sólido. Tesis de Doctorado, Cuba (2005).

Frazier, W.C y D.C. Westhoff; Microbiología de los Alimentos, p. 681, Ed. Acribia, Zaragoza, España (2000).

Gibson, A.M. y A.D. Hocking; Advances in the predictive modeling of fungal growth in food. Trends Food Sci. Technol., 8, 353-358 (1997).

Jay, J.M; Microbiología moderna de los Alimentos, 4ta. Ed. Ed. Acribia, Zaragoza, España (2002).

McMeekin, T.A., T. Ross, J. Oley y D.A. Ratkowsky; Predictive microbiology. Theory and application, 59-70. John Wiley and Sons, New York, USA (1993).

Meletiadis, J., J.F.G.M. Meis, J.W. Mouton y P.E. Verweij; Analysis of growth characteristics of filamentous fungi in different nutrient media. J. Clin. Microbiol., 39, 478-484 (2001).

Norrish, R.S.; An equation for the activity coefficients and equilibrium relative humidities of water in confectionary syrups, J. Food Technol., 1, 25 (1966).

Panagou, E.Z., P.N. Skandamis, y G.J.E. Nychas; Modelling the combined effect of temperature, $p H$ and $\mathrm{a}_{w}$ on the growth rate of Monascus ruber, a heat-resistant fungus isolated from green table lives, J. Appl. Microbiol., 94(1), 146-156 (2003).

Ross, K.; Estimation of water activity in intermediate moisture foods. Food Technol. 29(3), 26-30 (1975).

Samson, R., E. Hoekstra, J. Frisvad y O., Filtenborg; Introduction to Food Borne Fungi, 20-21. $4^{\mathrm{a}}$ ed. Centraalbureau Voor Schimmelcultures. The Netherlands (1995).

Shapira, R. y otros cinco autores; Detection of aflatoxigenic molds in grains by PCR, Appl. Environ. Microbiol., 62 (9), 3270-3273 (1996).

Vereecken, K.M., E.J. Dens y J.F. Van Impe; Predictive modeling of mixed microbial populations in food products: evaluation of two-species models, J. Theoret. Biol. 205 (1), 53-72 (2000).

Xiong, R. y otros cuatro autores; Comparison of the Baranyi model with the modified Gompertz equation for modeling thermal inactivation of Listeria monocytogenes Scott A, Food Microbiol., 16(3), 269-279 (1999). 